\title{
Annual acknowledgement of manuscript reviewers
}

Alexander Tenenbaum ${ }^{1 *}$ and Enrique Fisman ${ }^{2}$

\section{Contributing reviewers}

The Editors of Cardiovascular Diabetology wish to thank all reviewers, both Editorial Board Members and external referees, who contributed to the journal in 2012 (Volume 11), and whose enthusiastic support in providing the essential service of peer review continues to improve the scientific quality of the journal and enable its goals to be achieved.

\author{
Mehrshad Abbasi \\ Iran \\ Pasquale Abete \\ Italy \\ Hitoshi Adachi \\ Japan
}

Carlos A. Aguilar Salinas

Mexico

Aila Ahola
Finland

Yoshifusa Aizawa

Japan

Yasuo Akanuma

Japan

Rajadurai Akilen

United Kingdom

Nasser Al-Daghri

Saudi Arabia

Nikolaos Alexopoulos

Greece

Zaid Hezam Al-Hamodi

Malaysia

Mouaz Al-Mallah

Saudi Arabia

\author{
Amy Alman \\ United States of America
}

Alexandra Alvarsson

Sweden

Tetsuya Amano

Japan

Giuseppe Ambrosio

Italy

Charlotte Andersson

Denmark

Christian Anderwald

Austria

Ioanna Andreadou

Greece

Daniele Andreini

Italy

Francesco Andreozzi

Italy

Monica Andrews

Chile

Hidenori Arai

Japan

Hisatomi Arima

Australia
Benoit Arsenault
Netherlands

Kei Asayama

Belgium

Yoshimasa Aso

Japan

Kirsi Auro

Finland

Pablo Avanzas

Spain

Vajreswari Ayyalasomayajula India

\section{Gopal Babu}

United States of America

Ljuba Bacharova

Slovakia

Richard Bagnall

United Kingdom

Christopher Baines

United States of America

Enzo Ballotta

Italy

Yongping Bao

United Kingdom

\footnotetext{
* Correspondence: altenen@yahoo.com

${ }^{1}$ Cardiac Rehabilitation Institute, Sheba Medical Center, 52621, Tel-Hashomer, Israel

${ }^{2}$ Sackler Faculty of Medicine, Tel-Aviv University, 69978, Tel-Aviv, Israel
}

\section{() Biomed Central}

(c) 2013 Tenenbaum and Fisman; licensee BioMed Central Ltd. This is an Open Access article distributed under the terms of the Creative Commons Attribution License (http://creativecommons.org/licenses/by/2.0), which permits unrestricted use, distribution, and reproduction in any medium, provided the original work is properly cited. 
Elizabeth Barrett-Connor

United States of America

Rene Baudrand

Chile

John Baynes

United States of America

Abdulbari Bener
Qatar

Don Benjamin

Switzerland

Joline Beulens

Netherlands

Michel Beylot

France

Dwaipayan Bharadwaj

India

Yochai Birnbaum

United States of America

Simona Bo

Italy

Mona Boaz

Israel

Leandro Boer-Martins

Brazil

Steffen Bohler

Germany

L Cornelius Bollheimer

Germany

Fabrice Bonnet

France

Carlo Briguori

Italy

Carlos Brotons

Spain

Christa Buechler

Germany

Clay Bunn

United States of America

Monika Buraczynska

Poland

Christof Burgdorf

Germany

Francesco Cacciatore

Italy

\author{
Stefano Cagnin \\ Italy \\ Maria Calatayud \\ Spain \\ Duncan Campbell \\ Australia \\ Massimiliano Caprio \\ Italy \\ Françoise Carlotti \\ Netherlands \\ Axel Carlsson \\ Sweden \\ Jan Cederholm \\ Sweden \\ Piero Ceriana \\ Italy \\ Rajesh Chaudhary \\ Thailand \\ Wei Chen \\ United States of America \\ Mien-Cheng Chen \\ Taiwan \\ Jaw-Wen Chen \\ Taiwan \\ Hsing I Chen \\ Taiwan \\ Brian Chen \\ United States of America \\ Shiyou Chen \\ United States of America \\ Min Cheng \\ China \\ Hoi Lun Cheng \\ Australia \\ Juei-Tang Cheng \\ Taiwan \\ Adnan Chhatriwalla \\ United States of America \\ Robert Chilton \\ United States of America \\ Jaye Chin-Dusting \\ Australia \\ Tak-Shing Ching \\ Taiwan
}

Sungsam Cho

Japan

Zhao Zhong Chong

United States of America

Nain-Feng Chu

Taiwan

Peter Chudý

Slovakia

Arrigo FG Cicero

Italy

Iwona Cicha

Germany

Emmanuel Cosson

France

Stuart Craig

United States of America

Cora Cymeryng

Argentina

Wangde Dai

United States of America

Andreas Daiber

Germany

Robert Dalla Pozza

Germany

Paresh Dandona

United States of America

Merav Darash-Yahana

Israel

Michael Dashwood

United Kingdom

Wendy Davis

Australia

Dirk De Bacquer

Belgium

Peter Wilhelmus de Leeuw

Netherlands

Richard Dearborn Jr.

United States of America

Christi Deaton

United Kingdom

Mark DeBoer

United States of America

M Isabel del Olmo

Spain 


\begin{tabular}{|c|c|c|}
\hline Aldona Dembinska-Kiec & Michiaki Fukui & Matthew Gurka \\
\hline Poland & Japan & United States of America \\
\hline Patrick Dessein & Tohru Funahashi & Ida Gustafsson \\
\hline South Africa & Japan & Denmark \\
\hline George Dimitriadis & Martha Funnell & Anne Hafstad \\
\hline Greece & United States of America & Norway \\
\hline Hong Ding & Masato Furuhashi & Ganesh Halade \\
\hline Qatar & Japan & United States of America \\
\hline Ellen Dirkx & Trudy Gaillard & Samar M. Hammad \\
\hline Belgium & United States of America & United States of America \\
\hline Dragan Djuric & Xin Gao & Juying Han \\
\hline Serbia & China & Canada \\
\hline Sergio Dubner & Luis García-Ortiz & Scott Harding \\
\hline Argentina & Spain & United Kingdom \\
\hline Reinhard Duehlmeier & William Garver & Fatma Harzallah \\
\hline Germany & United States of America & Tunisia \\
\hline Jacques Dumont & Irene Gazi & Goji Hasegawa \\
\hline Belgium & Greece & Japan \\
\hline Kensuke Egashira & Zhi-Dong Ge & Yuichi Hattori \\
\hline Japan & United States of America & Japan \\
\hline Kai Eggers & Adriana Georgescu & Toshio Hayashi \\
\hline Sweden & Romania & Japan \\
\hline Robert Elkeles & Cosimo Giannini & Zeliha Hekimsoy \\
\hline United Kingdom & United States of America & Turkey \\
\hline Jackie Elliott & Bruna Gigante & Hans-Werner Hense \\
\hline United Kingdom & Sweden & Germany \\
\hline Mehmet Erdogan & Janaina Gonçalved & Michel P Hermans \\
\hline Turkey & Brazil & Belgium \\
\hline Serpil Eroglu & Jørgen Gravning & Ramon Hermida \\
\hline Turkey & Norway & Spain \\
\hline David Faeh & Jacoba P Greving & Hiroshi Hirose \\
\hline Switzerland & Netherlands & Japan \\
\hline Giuseppe Faggian & Garrett Gross & Marion Hofmann \\
\hline Italy & United States of America & United States of America \\
\hline Inês Falcão-Pires & Bruno Guerci & Martin Hohenegger \\
\hline Portugal & France & Austria \\
\hline Britt Falskov & Zhixin Guo & Philip Home \\
\hline Denmark & China & United Kingdom \\
\hline Csaba Farsang & Hua Guo & Dongming Hou \\
\hline Hungary & United States of America & United States of America \\
\hline John Feiner & Alok Kumar Gupta & Peng $\mathrm{Hu}$ \\
\hline United States of America & United States of America & China \\
\hline Trevor Ferguson & Alev Gurgun & Qiaobing Huang \\
\hline Jamaica & Turkey & China \\
\hline
\end{tabular}


Yu Huang

China

Abdelaziz Hussein
Egypt

Chii-Min Hwu

Taiwan

Iskandar Idris

United Kingdom

Satoshi Ikeda

Japan

Ignatios Ikonomidis

Greece

Wilfred Ip

Canada

Masaharu Ishihara

Japan

Yasuhiro Ishihara

Japan

Nobukazu Ishizaka

Japan

Nobuhiko Ishizuka

Japan

Milosz Jaguszewski

Switzerland

Karin Jandeleit-Dahm

Australia

Myung Ho Jeong

Korea, South

Gyorgy Jermendy

Hungary

Lin Jiang

United States of America

Manuel Jimenez-Navarro

Spain

Odd Erik Johansen

Norway

Brent Johnston

Canada

Michael Joner

Germany

Chan Hee Jung

South Korea

Markus Juonala

Finland
Satomi Kagota

Japan

Radhika Kakarala

United States of America

Mohd Fozi Kamarudin

Malaysia

Masahiro Kamouchi

Japan

Ippei Kanazawa

Japan

Raj Kandpal

United States of America

Hideaki Kaneto

Japan

Tom Karagiannis

Australia

Jens Kastrup

Denmark

Naoto Katakami

Japan

Akihiko Kato

Japan

Ryuichi Kawamoto

Japan

Malte Kelm

Germany

Mykola Khalangot

Ukraine

Haseeb Khan

Saudi Arabia

Zia Khan

Canada

Ahsan Khandoker

Australia

Madhu Khullar

India

Daejung Kim

South Korea

Shokei Kim-Mitsuyama Japan

Klaus-Dieter Kohnert

Germany

Pappachan Kolattukudy

United States of America
Malcolm Koo

Canada

Magdalena Kostkiewicz

Poland

Masoumeh Kourosh Arami

Japan

Irina Kowalska

Poland

Detlef Kozian

Germany

Marek Kozinski

Poland

Rungroj Krittayaphong

Thailand

Wiktor Kuliczkowski

Poland

Hemant Kulkarni

United States of America

Adnan Kurdal

Turkey

Claudia Kusmic

Italy

Johanna Kuusisto

Finland

Andreas Kuznik

United States of America

Veronique Lacombe

United States of America

Jorma Lahtela

Finland

Tomi Laitinen

Finland

Wendy Lane

United States of America

Cia-Hin Lau

Malaysia

Harold Lazar

United States of America

Tennille Leak-Johnson

United States of America

Verna Lee

Malaysia

Won Young Lee

Korea, South 
Byung-Wan Lee

Korea, South

Yong-Jae Lee

Korea, South

I-Te Lee

Taiwan

Michael Lehrke

Germany

Ioannis Lekakis

Greece

Konstantinos Letsas

Greece

Andrew Levy

Israel

Kae-Woei Liang

Taiwan

Wei Liang

China

Yi-Chu Liao

Taiwan

Atip Likidlilid

Thailand

Kuan Pin Lin

Taiwan

Michael Lipinski

United States of America

Que Liu

United States of America

Bicheng Liu

China

Brian Bridal Løgstrup

Denmark

Gary Lopaschuk

Canada

Carolina Lourenço

Portugal

Hongyun Lu

China

Stephan Lüders

Germany

Olga Lunegova

Kyrgyzstan

Christoph Maack

Germany
Imad Maatouk

Germany

Stephen Macdonald

Australia

Rogelio Alberto Machado

Argentina

Agnieszka Madro

Poland

Shiro Maeda

Japan

Sujata Mahadik

India

Atheline Major-Pedersen

Denmark

Safarina Golfiani Malik

Indonesia

Kai Mao

United States of America

Pedro J Marín

Spain

Raffaele Marfella

Italy

Jennifer Martin

Australia

Francisco Javier Martinez-Martin

Spain

Raul Martins

Portugal

Masafumi Matsuda

Japan

Laetitia Mauge

France

Helen McDonald

United Kingdom

Jane McElroy

United States of America

Philip McTernan

United Kingdom

Manoj Medal

India

Patrick Meimoun

France

Christa Meisinger

Germany
Jordi Merino

Spain

Patricia Metcalf

New Zealand

Takayuki Miki

Japan

Masoud Mirzaei

Australia

Toru Miyoshi

Japan

Denise Momesso

Brazil

Louis Monnier

France

Monica Montagnani

Italy

Ryuichi Morishita

Japan

Yusuke Moritoh

Japan

Antonio Muscari

Italy

Elza Muscelli

Italy

Fawaz Mzayek

United States of America

Sath Nag

United Kingdom

Shashidhar Nagaraj

India

Kei Nakajima

Japan

Manouchehr Nakhjavani

Iran

Emilio Nardi

Italy

Loredan Stefan Niculescu

Romania

Peter Nilsson

Sweden

Peter Nilsson

Sweden

Koichi Node

Japan 


\author{
Ryuji Nohara \\ Japan
}

Anna Norhammar

Sweden

Mads Nybo

Denmark

Anders Nygren

Canada

Mark O'Donnell

United Kingdom

Hiroshi Ogawa

Japan

Hisao Ogawa

Japan

Robert Ohsfeldt

United States of America

Yosuke Okada

Japan

Satoshi Okayama

Japan

Sukma Oktavianthi

Indonesia

Silvio Oliveira Junior

Brazil

Trine Baur Opstad

Norway

Emilio Ortega

Spain

Alfonso Otero Gonzalez Spain

Noriyuki Ouchi

Japan

Nobuaki Ozaki

Japan

Pal Pacher

United States of America

Raluca Pais

France

Barry Palmer

New Zealand

Yang Pan

United States of America

Dimitrios Papadopoulos

Greece
Ioannis Papassotiriou

Greece

Athanasios G. Papavassiliou

Greece

Cheol-Young Park

South Korea

Hyeong-Kyu Park

South Korea

Joong-Yeol Park

South Korea

Anthony Passerini

United States of America

Ulrik Pedersen-Bjergaard

Denmark

Michael Pencina

United States of America

Wenhui Peng

China

Grant Pierce

Canada

Antonio Pinto

Italy

Dario Pitocco

Italy

Bertram Pitt

United States of America

Michael Poteser

Austria

Fabrice Prunier

France

Tran Quang Binh

Vietnam

Edward Randell

Canada

Ankit Rathod

United States of America

Itamar Raz

Israel

Peter Reaven

United States of America

Flavio Reis

Portugal

Alan Remaley

United States of America
Jun Ren

United States of America

Markus Resch

Germany

Fausto Rigo

Italy

Stefano Rimoldi

Switzerland

Maria Rosaria Rizzo

Italy

Testa Roberto

Italy

Bruno Rodrigues

Brazil

Martha Rodriguez-Moran

Mexico

Robert Rosenson

United States of America

Ina-Maria Rückert

Germany

Sandeep Saha

United States of America

M Ram Sairam

Canada

Masaya Sakamoto

Japan

Naoki Sakane

Japan

Juha Saltevo

Finland

Rafael Salto

Spain

Erik Salum

Estonia

Paulo Santos

Brazil

Gaetano Santulli

United States of America

Ayhan Saritas

Turkey

Kevin Schalinske

United States of America

Arthur Scholte

Netherlands 


\author{
Aletta Schutte \\ South Africa \\ Karl Schwab \\ Germany \\ Neal Scott \\ United States of America \\ Fernando Segade \\ United States of America \\ Yutaka Seino \\ Japan
}

Ken-ichi Serizawa

Japan

Amir Shafat

Ireland

Michal Shani

Israel

Jayashree Shanker

India

Sushma Sharma

United States of America

Sunil sharma

India

Michael Shechter

Israel

Weifeng Shen

China

Wayne Sheu

Taiwan

Zumin Shi

Australia

Rei Shibata

Japan

JaeYong Shim

Korea, South

Toshio Shimada

Japan

Kazunori Shimada

Japan

Tetsuro Shishido

Japan

Manisha Shrivastav

United States of America

David Simmons

United Kingdom
Scot Simpson

Canada

Vivek Singh

United States of America

Susan Sisson

United States of America

Sandhya Sitasawad

India

Ivonne Sluijs

Netherlands

Irina Smirnova

United States of America

Andries Smit

Netherlands

Janet Snell-Bergeon

United States of America

Elsayed Z. Soliman

United States of America

Maria de Fatima Sonati

Brazil

Dinesh Kumar Srinivasan

Singapore

Eberhard Standl

Germany

Arie Steinvil

Israel

Stephanie Stock

Germany

Joshua Stolker

United States of America

Celia Strunz

Brazil

Milton Suarez-Ortegón

Colombia

Elena Succurro

Italy

Takayoshi Suganami

Japan

J David Symons

United States of America

Cheuk Chun Szeto

Hong Kong

Yasuharu Tabara

Japan
Heinrich Taegtmeyer

United States of America

Isao Taguchi

Japan

Julio Takada

Brazil

Yasuo Takahashi

Japan

Takeshi Takami

Japan

Kumiko Takemori

Japan

Junichi Taki

Japan

Charmaine Tam

Australia

Luosheng Tang

China

Giovanni Targher

Italy

Ulrich Tebbe

Germany

Alexander Tenenbaum

Israel

Nicholas Tentolouris

Greece

Vicki Thallas-Bonke

Australia

Stine Thomsen

Denmark

Stephen Thomson

United States of America

Chengju Tian

United States of America

Ivan Tkac

Slovakia

Hirofumi Tomiyama

Japan

Arpad Tosaki

Hungary

Dimitris Tousoulis

Greece

Antonino Tuttolomondo Italy 


Shiro Uemura
Japan
Matti Uusitupa
Finland
Rodolfo Valdez
United States of America
Serafina Valente
Italy
Charissa van den Brom
Netherlands
Ingrid van der Mei
Australia
Mariska van Vliet
Netherlands
Luca Vanella
Italy
Keri Vartanian
United States of America
Elena Velkoska
Australia
Marianne Verhaar
Netherlands
Suman Verma
India
Richard Verrier
United States of America

Saula Vigili de Kreutzenberg Italy

\section{Anders Vik}

Norway

Jeffrey Voigt

United States of America

Adriaan Voors

Netherlands

Christina Voulgari

Greece

Brian Walker

United Kingdom

Yujie Wang

United States of America
Xin Wang

United Kingdom

Wei Wang

United States of America

Wuan-Szu Wang

Taiwan

Marie-Louise Ward

New Zealand

G. Russell Warnick

United States of America

Anna Watson

Australia

Julian Widder

Germany

Robert Wilensky

United States of America

Jenifer Wogen

United States of America

\section{Cheuk-Kit Wong}

New Zealand

Wen-Bin Wu

Taiwan

Yi Wu

United States of America

Xunde Xian

United States of America

Liang Xiao

China

Huang Xienan

China

Ya-Wei Xu

Colombia

Shiming Xu

United States of America

Susan Xu

United States of America

Biao Xu

China

Daisuke Yabe

Japan
Neerja Yadav

India

Kazuhiro Yamamoto

Japan

Koichi Yamamoto

Japan

Yi-Ling Yang

Taiwan

Lina Yang

United States of America

Zhen Yang

China

Bu Yeap

Australia

Joseph Yeboah

United States of America

Fawn Yeh

United States of America

Kai Hang Yiu

Hong Kong

Hiroshi Yoshida

Japan

Xinhua Yu

United States of America

Han-Gang Yu

United States of America

Markus Zabel

Germany

Feng Zhang

United States of America

Giacomo Zoppini

Italy

Yunzeng Zou

China

Chenhui Zou

United States of America

Giovanni Zuliani

Italy

doi:10.1186/1475-2840-12-43

Cite this article as: Tenenbaum and Fisman:

Annual acknowledgement of manuscript

reviewers. Cardiovascular Diabetology 2013

12:43. 